reveals various clinical course and therapeutic responsiveness according to the clinical and serological subsets. Some myositis-specific autoantibodies (MSAs) are useful markers for the classification of ILD in myositis and give useful information for predicting the prognosis and determining treatment.

Anti-aminoacyl-tRNA synthetases are closely associated with a common clinical manifestation, termed "anti-synthetase syndrome" including high prevalence of ILD. ILD in patients with anti-synthetases shows a similar clinical course with a favourable response to therapy but frequent recurrences. Therefore, the concomitant use of glucocorticoids and immunosuppressive drugs is recommended from early stage of the disease.

Anti-MDA5 antibody was reported to be associated with clinically amyopathic dermatomyositis (CADM) with rapidly progressive ILD, especially in eastern Asian population. Because of a very poor life prognosis, the intensive immunosuppressive therapy against ILD from early stage is recommended using the combination of high dose glucocorticoids, calcineurin inhibitors and intravenous cyclophosphamide. We have experienced the effectiveness of plasmapheresis in some anti-MDA-positive patients with intractable ILD, suggesting a possible pathogenicity of anti-MDA5 antibody.

Thus, ILD in myositis is dependent on the autoantibodies, therefore it is important to know the profiles of MSAs in PMDM patients. Recently, we established the ELISA systems for anti-synthetase and anti-MDA5 antibodies, which are as efficient as the standard immunoprecipitation assays. These systems enable easier and wider use in the diagnosis and therapeutic decision of patients suspected to have myositis and ILD.

\section{THE INTERNATIONAL CONSENSUS ON STANDARDIZED NOMENCLATURE OF ANTINUCLEAR ANTIBODY HEP-2 CELL PATTERNS (ICAP) INITIATIVE - ITS IMPACT AND UPDATE FROM 3RD ICAP}

${ }^{1} \mathrm{E}$ Chan*, 2J Damoiseaux, OG Carballo ${ }^{3},{ }^{4} \mathrm{~K}$ Conrad, ${ }^{5} \mathrm{~W}$ de Melo Cruvinel, PLCFrancescantonio ${ }^{6}{ }^{7} \mathrm{M}$ Fritzler, ${ }^{8}$ I Garcia-De La Torre, ${ }^{9} \mathrm{M}$ Herold, ${ }^{10} \mathrm{~T}$ Mimori, ${ }^{11} \mathrm{M}$ Satoh, ${ }^{12} \mathrm{C}$ von Mühlen, ${ }^{13} \mathrm{~A}$ Luis. ${ }^{1}$ USA; ${ }^{2}$ Maastricht University Medical Centre, Central Diagnostic Laboratory, Maastricht, The Netherlands; ${ }^{3}$ Hospital Carlos G. Durand, Laboratory of Immunology, Buenos Aires, Argentina; ${ }^{4}$ Technical University of Dresden, Institute of Immunology, Dresden, Germany; ${ }^{5}$ Pontificia Universidade Católica de Goiás, Biology, Goiânia, Brazil; ${ }^{6}$ Pontifícia Universidade Católica de Goiás, Medicine, Goiânia, Brazil; 'University of Calgary, Medicine, Calgary, Canada; ${ }^{8}$ University of Guadalajara, Immunology and Rheumatology, Guadalajara, Mexico; ${ }^{9}$ Medical University of Innsbruck, Internal Medicine VI, Innsbruck, Austria; ${ }^{10}$ Kyoto University, Rheumatology and Clinical Immunology, Kyoto, Japan; ${ }^{11}$ University of Occupational and Environmental Health, Clinical Nursing, Kitakyushu, Japan; ${ }^{12}$ Brazilian Society of Autoimmunity, Immunology, Porto Alegre, Brazil; ${ }^{13}$ Universidade Federal de São Paulo, Rheumatology, Sao Paulo, Brazil

10.1136/lupus-2017-000215.27

Background and Aims The indirect immunofluorescence (IIF) pattern in ANA test provides initial assessment of autoantibody responses in candidate patients at risk of developing SLE. The International Consensus on ANA Patterns (ICAP) Initiative workshop originated in 2014, followed by the $2^{\text {nd }}$ and $3^{\text {rd }}$ workshops in 2015 and 2016. The goal of ICAP is to provide guidelines for ANA interpretation, thereby optimising adoption in diagnostic laboratories and extended to patient care.

Methods A working committee addressed collective issues on ANA nomenclature that are raised by participants representing research, clinical, and diagnostic laboratories.

Results ANA patterns are separated into three major categories (nuclear, cytoplasmic, and mitotic patterns). A total of 28 patterns were defined, described in detail, designated with alpha-numeric codes (AC-1 to AC-28), and summarised under a nomenclature and classification tree (ANApatterns.org). ICAP initiatives include translation of the website into other languages, establishing guidelines in ANA reporting, and programs for continuing education. The translation initiative promotes the establishment and dynamic engagement of a worldwide network. To date, the website displays its content in English, Spanish, Portuguese, Italian and German, while Chinese, Japanese, French, and Greek translations are ongoing. ICAP has provided a common platform to address issues that are of great interest to the scientific community. The establishment of a consensus on ANA reporting will require interaction with committees in charge of establishing disease classification and diagnostic criteria.

Conclusions Future goals include building collaborative data on ANA patterns, establishing new consensus patterns, and developing an interpretative description for each pattern for their widespread clinical use.

\section{DRIVERS OF THE SLE RESPONDER INDEX (SRI-5) ENDPOINT IN CLINICAL TRIALS OF SLE}

${ }^{1} \mathrm{~K}$ Kalunian, ${ }^{*}{ }^{2} \mathrm{M}$ Urowitz, ${ }^{3} \mathrm{D}$ Isenberg, ${ }^{4} \mathrm{~J}$ Merrill, ${ }^{5} \mathrm{M}$ Petri, ${ }^{6} \mathrm{R}$ Furie, MA Morgan$\mathrm{Cox}^{7},{ }^{7} \mathrm{R}$ Taha, ${ }^{8} \mathrm{M}$ Silk, ${ }^{9} \mathrm{M}$ Linnik. ${ }^{1}$ University of California- San Diego School of Medicine, Medicine, San Diego, USA; ${ }^{2}$ University of Toronto Faculty of Medicine, Medicine, Tornoto, Canada; ${ }^{3}$ University College London, Rheumatology, London, UK; ${ }^{4}$ University of Oklahoma College of Medicine, Rheumatology, Oklahoma City, USA; ${ }^{5}$ Johns Hopkins University School of Medicine, Rheumatology, Baltimore, USA; ${ }^{6}$ Hofstra North Shore-LIJ School of Medicine, Rheumatology, Great Neck, USA; ' Eli Lilly, Statistics, Indianapolis, USA; ${ }^{8}$ Eli Lilly, Immunology, Indianapolis, USA; ' ${ }^{2}$ illy Biotechnology Centre, Immunology, San Diego, USA

\subsection{6/lupus-2017-000215.28}

Background and Aims SRI is a composite endpoint used in many SLE trials. The current investigation sought to identify those SRI components that drive response.

Methods This study evaluated data from two large multinational trials that used SRI-5 as primary endpoint $(n=2262$ patients).

Results The overall SRI-5 response rate was 32.8\%. Nonresponse due to lack of a 5-point SLEDAI improvement, conmed violation or dropout were observed in 31\%, 16.5\% and $19.1 \%$, respectively. In contrast, only $0.5 \%(11 / 2262)$ of patients failed to respond due to deterioration by BILAG or PGA, once achieving the first three components.

The most common SLEDAI organ systems involved at baseline were mucocutaneous (90.6\%), musculoskeletal (82.9\%) and immunologic $(71.6 \%)$. The 6 other SLEDAI-defined organ systems were active in $\leq 11 \%$ of patients.

At least one SLEDAI manifestation with low prevalence and high score ( $\geq 4$ pts) was present in $18.1 \%$ and $17.2 \%$ of patients in Trials 1 and 2, respectively. SRI-5 response rates for these patients were higher, regardless of treatment assignment.

Conclusions The SRI-5 response is driven by SLEDAI improvement, concomitant medication adherence and trial completion. Patients with less frequent, more severe manifestations had high placebo response rates. A simple dichotomous improvement in SLEDAI score, coupled with successful trial completion and medication stability, provides a more efficient, clinically relevant approach to assess outcome. 


\section{Parallel Session 10: APS}

\section{ANTIPHOSPHOLIPID SCORE IS A NOVEL RISK FACTOR FOR IDIOPATHIC OSTEONECROSIS}

N Ohnishi*, M Kato, Y Ogata, S Abe, M Kono, Y Shibata, S Tanimura, R Hisada, M Doi, Y Fujieda, K Oku, T Bohgaki, O Amengual, S Yasuda, T Atsumi. Hokkaido University Graduate School of Medicine, Division of Rheumatology- Endocrinology and Nephrology, Sapporo, Japan

\subsection{6/lupus-2017-000215.29}

Background and Aims Our group introduced a quantitative marker of antiphospholipid antibodies (aPL) "antiphospholipid score (aPL-S)", which well reflected the risk of developing thrombosis (Otomo K, et al. Arthritis Rheum 2012). Idiopathic osteonecrosis (ION) has been shown to occur as a result of ischemia, however, the involvement of aPL in its pathophysiology remains to be elucidated. In this study, we aimed to identify the impact of aPL on the development of ION using aPL-S.

Methods A single centre retrospective study comprising 75 consecutive patients with systemic lupus erythematosus who underwent magnetic resonance imaging of hip joints from January 2000 to March 2016. aPL-S, as well as classical risk factors for ION, were evaluated in all the enrolled patients.

Results ION of the femoral head was observed in 33 out of 75 patients(44\%). High aPL-S $(\mathrm{p}=0.009)$, aPL positivity $(\mathrm{p}=0.009)$, male $(\mathrm{p}=0.007)$, malar rash $(\mathrm{p}=0.010)$ and high dose $(>0.8 \mathrm{mg} / \mathrm{kg} /$ day $)$ glucocorticoids $(\mathrm{p}<0.001)$ were identified as risk factors for ION at univariate analysis. Multivariate analysis confirmed high aPL-S, male, malar rash and high dose glucocorticoids as independent variables. Six out of 8 patients $(75 \%)$ with very high aPL-S (>30), developed ION. Conversely, systemic lupus erythematosus disease activity index and pulse methylprednisolone therapy were not identified as risk factors for ION.

Conclusions We newly identified aPL-S as a risk factor for ION. ION should be considered as one of the antiphosholipid antibody-associated-disease.

\section{Parallel Session 11: Effector T cells in SLE}

\section{METABOLIC REPROGRAMMING IN CD4+CD28- CXCR3INTT-BETHI CELLS AND ITS RELEVANCE TO PATHOGENESIS IN PATIENTS WITH SLE}

\begin{abstract}
${ }^{1} \mathrm{~S}$ Iwata*, 'K Sakata, ${ }^{1} \mathrm{M}$ Hajime, ${ }^{1} \mathrm{M}$ Zhang, ${ }^{1,2} \mathrm{M}$ Torigoe, ${ }^{1} \mathrm{~N}$ Ohkubo, ${ }^{1} \mathrm{~S}$ Nakayamada, ${ }^{1} Y$ Tanaka. 'University of Occupational and Environmental Health, The First Depertment of Internal Medicine, Kitakyushu, Japan; ${ }^{2}$ Department of Endocrinology- MetabolismRheumatology and Nephrology, Faculty of Medicine- Oita University- Yufu- Oita- Japan, Yufu, Japan
\end{abstract}

\subsection{6/lupus-2017-000215.30}

Background and Aims $\mathrm{CD}^{+} \mathrm{T}$ cells play a crucial role in pathological process of Systemic Lupus Erythematosus (SLE). Recently, we found that T-bet is an important factor for shift to glycolysis in activated $\mathrm{CD}^{+}{ }^{+} \mathrm{T}$ cells in vitro. In this study, we examined the mechanism by which T-bet in $\mathrm{CD}^{+} \mathrm{T}$ cells involved in pathogenesis of SLE.
Methods Peripheral blood mononuclear cells were obtained from 19 healthy controls (HCs), 30 patients with bio-naïve active RA and 60 patients with SLE. The expression of CXCR3, T-bet, mTORC1 phosphorylation and IFN- $\gamma$ production in $\mathrm{CD}^{+} \mathrm{T}$ cells were measured by flow cytometry, and assessed the association with clinical characteristics.

Results We found that the ratio of $\mathrm{CD} 28^{-} \mathrm{CXCR} 3^{\text {int }} \mathrm{T}-\mathrm{bet}{ }^{\text {hi }}$ cells in patients with SLE was significantly higher compared to HCs. CD $4{ }^{+} \mathrm{CD} 28^{-} \mathrm{CXCR} 3^{\text {int }}$ T-bet ${ }^{\text {hi }}$ cells mainly consisted of CD45RA ${ }^{-}$CCR7 ${ }^{-}$effector memory cells and were significantly activated with pronounced IFN- $\gamma$ production. Interestingly, the ratio of $\mathrm{CD} 4^{+} \mathrm{CD} 28^{-} \mathrm{CXCR} 3^{\text {int }}{ }^{\mathrm{T}}$-bet ${ }^{\text {hi }}$ cells was significantly correlated with the number of immunosuppressants previously used for the SLE patients, that is treatment-resistant. Phosphorylation of mTORC1, which is important for shift to glycolysis, in $\mathrm{CD}^{+} \mathrm{T}$ cells from patients with SLE was significantly increased compared to HCs. T-bet expression was significantly correlated with mTOC1 phosphorylation and IFN- $\gamma$ production in $\mathrm{CD}^{+}{ }^{+} \mathrm{T}$ cells from patients with SLE.

Conclusions These results indicated that $\mathrm{CD} 4{ }^{+} \mathrm{CD} 28^{-} \mathrm{CXCR}^{\text {int }}{ }^{\mathrm{T}}$-bet ${ }^{\text {hi }}$ cells might be related to refractory to established therapies in patients with SLE. In addition, these cells are constitutively activated accompanied with shift to glycolysis through IFN- $\gamma$-mTORC1-T-bet pathway, which is a potential target for patients with SLE.

\section{Parallel Session 15: Pearls in autoimmunity}

\section{HARNESSING AUTOIMMUNITY (DISEASE-SPECIFIC AUTOANTIBODY AND ITS VARIANT) IN THERANOSTICS OF DISEASE \\ ${ }^{1}$ E Matsuura*. 'Okayama University, Collaborative Research Centre and Department of Cell Chemistry, Okayama, Japan}

\subsection{6/lupus-2017-000215.31}

The prevalence of autoimmune disorder or disease is characterised by its targeting autoantibodies. The term "theranostic", an innovative concept of medical modality featuring a portmanteau of therapeutic and diagnostic systems, was coined in 2002 and has since undergone progressive development into current preclinical stages. Recently, we have prepared humanised and shortened variants of IgG (single chain variable fragment; $25 \mathrm{kDa}-\mathrm{scFv}$ ) targeting towards: (i) $\beta 2$-glycoprotein I ( $\beta 2$-GPI) complexed with oxidised LDL, a key population of pathogenic autoantibodies related to the development of antiphospholipid syndrome (APS) and autoimmune mediated atherosclerosis, and (ii) mesothelin, a 40 kDa-tumour differentiation antigen, to establish a clinically applicable theranostic in autoimmune mediated atherosclerosis and oncology. Goals of our theranostic system (comprises of novel and biodegradable ${ }^{89} \mathrm{Zr}$-radiolabeled nanoparticles conjugated with specific scFv) are to successfully deliver therapeutically effective small interfering RNA (siRNA), for inducing apoptosis in targeted cells of experimental models and to offer simultaneous visualisation of the targets via PET imaging system. The combination of photodynamic therapy (PDT) with 5-aminolevulinic acid (ALA) and photo-controlled intracellular siRNA 